\title{
A Grounding System Model Directly in Time-Domain
}

\author{
C. M. Seixas and S. Kurokawa, Member, IEEE
}

\begin{abstract}
This work presents a technique for electrical grounding system representation, directly in the time domain. It is based on the theory of least squares and starts from harmonic grounding impedance, representing it by discrete element circuits (resistors, capacitors and inductors) associated in series-parallel. From the impedance can be determined the transient ground voltage due to current impulse (lightning) applied, which is one of the quantities of greatest interest in the grounding studies. The advantage of using models developed directly in the time domain is that they can be easily inserted into conventional simulation software, such as the Alternative Transient Program (ATP), Electromagnetic Transient Program (EMTP) and Orcad (PSPICE). To determine the harmonic grounding impedance ("view" from the point struck by lightning), was used as a tool a model based on the theory of transmission lines considering distributed parameters, but could be used any other model, independent of the simplifications and boundary conditions used in their representation, such as the electromagnetic model. The validation of this method was made by comparing the results of this work (for vertical rod and horizontal electrode) with another obtained and already validated through experimental results by one renowned research group in the electrical grounding area, achieving good agreement.
\end{abstract}

Keywords - Electrical grounding, electromagnetic transients, frequency domain, ground impedance, lightning, modeling, soil resistivity, time domain.

\section{INTRODUÇÃO}

$\mathrm{E}$ LETRODOS horizontais, hastes verticais, malhas de aterramento e complexas configurações de sistemas de aterramento são frequentemente usadas no sistema elétrico de potência e plantas industriais. $\mathrm{Na}$ ocorrência de descargas atmosféricas, a corrente impulsiva do surto flui pelo sistema de aterramento para ser dissipada no solo, podendo causar sobretensões no ponto de injeção desta corrente e em suas vizinhanças, bem como induzir tensões e provocar interferências eletromagnéticas em equipamentos conectados ao aterramento ou próximos a ele [1], [2]. Portanto, cuidados adequados devem ser tomados no projeto de sistemas de aterramento, especialmente em altas frequências (frequências típicas das descargas atmosféricas).

$\mathrm{Na}$ literatura técnica são encontradas diversas maneiras de representar sistemas de aterramento, baseadas nas teorias de circuitos elétricos [3], [4], de linhas de transmissão [5], [6] e de campos eletromagnéticos [7], [8]. Usando essas teorias, os modelos pioneiros foram elaborados empregando métodos

C. M. Seixas. Instituto Federal de São Paulo (IFSP), Votuporanga, Brasil. e Universidade Estadual Paulista (UNESP), Ilha Solteira, Brasil, claudiner2010@gmail.com.

S. kurokawa. Universidade Estadual Paulista (UNESP), Ilha Solteira, Brasil,kurokawa@dee.feis.unesp.br.

Corresponding author: Claudiner Mendes de Seixas. analíticos [9]-[11], métodos empíricos [12] e a partir da década de oitenta, devido aos avanços tecnológicos na área computacional, os métodos numéricos passaram a ser utilizados na grande maioria dos modelos. Os modelos ainda podem ser classificados como desenvolvidos no domínio do tempo ou da frequência. Segundo [13] e [14] os modelos baseados na teoria eletromagnética se fundamentam na resolução das equações de Maxwell e são mais precisos que os demais por apresentarem menos simplificações, porém suas implementações são mais complexa.

O objetivo deste trabalho é propor, aplicar e comprovar a eficácia de uma técnica desenvolvida para representar sistema de aterramento elétrico por um circuito elétrico equivalente, podendo este ser simulado diretamente no domínio do tempo por qualquer programa de computador destinado a este fim, dispensando o uso de transformada inversa de Laplace ou Fourier. A técnica é baseada no método de aproximação dos mínimos quadrados e está sendo aplicada neste trabalho a eletrodos horizontais e hastes verticais, porém conceito similar pode ser aplicado a qualquer configuração de sistema de aterramento, como, por exemplo, as malhas usadas em subestações, desde que seja fornecida a impedância harmônica de tal configuração. Pode também ser aplicada à torre de linha de transmissão, para representar sua impedância por circuitos elétricos, dentre outras aplicações, o que a torna muito interessante e versátil.

Devido sua simplicidade, facilidade de uso e por representar o eletrodo horizontal e a haste vertical com precisão adequada ao que se pretende mostrar, neste trabalho foi escolhido o modelo baseado na teoria de linhas de transmissão a parâmetros uniformemente distribuídos e independentes da frequência, não considerando o efeito da ionização do solo, para se determinar a impedância harmônica do aterramento. Qualquer outro modelo que forneça a impedância harmônica, independentemente das simplificações e condições de contorno utilizadas (modelos baseados ou não em aproximações quase estáticas, modelos a parâmetros dependentes ou não da frequência, modelos que levam ou não em consideração os efeitos da ionização do solo, da propagação e da interface solo-ar, modelos dinâmicos, etc), poderia ser usado e não interfere no método (mas sim nos resultados), visto que a técnica parte da impedância já determinada. Portanto a precisão do resultado dependerá do modelo escolhido e suas simplificações para determinação da impedância harmônica.

A técnica foi validada por meio de comparações dos resultados obtidos com os resultados de autores [14], [15] renomados na área de aterramento elétrico. Foi mostrada também a excelente concordância entre as curvas da tensão transitória obtida por meio do circuito RLC (circuito equivalente ao eletrodo de aterramento obtido via técnica proposta) e as curvas obtidas por meio do Universal Model 
Line (via modelo baseado na teoria de linha de transmissão a parâmetros distribuídos, tomado como padrão). Na seção II é apresentado o modelo baseado na teoria de linhas de transmissão utilizado para determinação da impedância harmônica dos eletrodos e hastes, na III é descrita a técnica proposta por este trabalho, na IV são apresentados os resultados obtidos e a validação e, na seção $\mathrm{V}$, a conclusão.

\section{MODELO BASEADO EM LINHAS DE TRANSMISSÃO}

Neste tópico descreve-se resumidamente o modelo baseado na teoria de linhas de transmissão (LT) a parâmetros uniformemente distribuídos. Ele tem sua origem nas equações diferenciais (1) e (2), conhecidas como equações do telegrafista, que possibilitam a determinação da tensão e da corrente em qualquer ponto ao longo de uma linha de transmissão, em função da distância “ $x$ ” [5], [16].

$$
\begin{aligned}
& \frac{d v(x, t)}{d x}=-R i(x, t)-L \frac{d i(x, t)}{d t} \\
& \frac{d i(x, t)}{d x}=-G v(x, t)-C \frac{d v(x, t)}{d t}
\end{aligned}
$$

As equações diferenciais, de difícil solução no domínio do tempo, podem ser resolvidas no domínio da frequência. Para tal, (1) e (2) podem ser reescritas conforme (3) e (4), sendo $Z=R+j \omega L$ a impedância longitudinal e $Y=G+j \omega C$ a admitância transversal da linha [16].

$$
\begin{aligned}
& \frac{d^{2} V(x, s)}{d^{2} x}=Z Y V(x, s) \\
& \frac{d^{2} I(x, s)}{d^{2} x}=Y Z I(x, s)
\end{aligned}
$$

As soluções de (3) e (4), no domínio da frequência, para uma haste ou eletrodo de comprimento "d" e considerando que a corrente em sua extremidade final é nula, é dada por (5) e (6), onde $Z_{c}=\sqrt{Z / Y}$ é a impedância característica e $\gamma=\sqrt{\mathrm{ZY}}$ é a função de propagação [14], [16].

$$
\begin{aligned}
& V_{\mathrm{A}}(s)=V_{\mathrm{B}}(s) \cosh (\gamma d) \\
& I_{\mathrm{A}}(s)=\frac{1}{Z_{c}} V_{\mathrm{B}}(s) \operatorname{senh}(\gamma d)
\end{aligned}
$$

A admitância harmônica pode ser escrita como sendo a relação entre o impulso de corrente e a elevação de tensão no ponto de aplicação do impulso, conforme (7).

$$
Y_{A}(s)=\frac{1}{Z_{c}} \operatorname{tgh}(\gamma d)
$$

O cálculo da admitância depende dos parâmetros (R, L, G e C) do conjunto eletrodo-solo e estes dependem da geometria e condutividade do eletrodo e da condutividade e permissividade elétrica do solo. As expressões para o cálculo destes parâmetros não foram apresentadas neste trabalho, mas estão disponíveis em vasta bibliografia como, por exemplo, em [5], [11], [14], [15].

\section{DESCRIÇÃO DA TÉCNICA PROPOSTA}

A técnica proposta consiste em aplicar a aproximação baseada no método dos mínimos quadrados (VF-Vector Fitting [17]) na curva da admitância harmônica $\left(\mathrm{Y}_{\mathrm{A}}\right)$ obtida pela função hiperbólica descrita em (7). Desta forma, obtémse a função racional que representa com fidelidade a curva da admitância, a partir da qual se determina o circuito elétrico equivalente que reproduz o mesmo efeito que a admitância.

O objetivo do VF é aproximar a resposta em frequência $\mathrm{F}$ (s) pela função racional, conforme (8), onde a função genérica $\mathrm{F}(\mathrm{s})$ é a admitância $\mathrm{Y}_{\mathrm{A}}(\mathrm{s}), D$ é o termo constante, $E$ é o termo dependente da frequência, $c_{i}$ são os resíduos, $a_{i}$ os polos e $s$ o operador igual a $j 2 \pi f$, sendo $f$ a frequência. Tanto os resíduos quanto os polos podem ser números reais ou complexos.

$$
F(s)=D+s E+\sum_{i=1}^{n} \frac{c_{i}}{s-a_{i}}
$$

Quando a fração parcial $\mathrm{F}_{\mathrm{R}}(\mathrm{s})$ possui resíduo e polo reais, conforme mostrado em (9), um possível circuito elétrico equivalente é apresentado na Fig. 1 [18].

$$
F_{R}(s)=\frac{c_{i}}{s-a_{i}}
$$

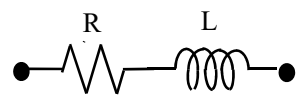

Figura 1. Circuito RL equivalente para resíduo e polo reais.

Neste caso os elementos de circuito R e L são calculados por (10) e (11).

$$
\begin{aligned}
& L=\frac{1}{c_{i}} \\
& R=-\frac{a_{i}}{c_{i}}
\end{aligned}
$$

Quando a fração parcial $\mathrm{F}_{\mathrm{C}}(\mathrm{s})$ possui resíduos e polos complexos conjugados, conforme (12), um possível circuito elétrico equivalente é apresentado na Fig. 2 [18]

$$
F_{C}(s)=\frac{c_{1}}{s-a_{1}}+\frac{c_{2}}{s-a_{2}}
$$

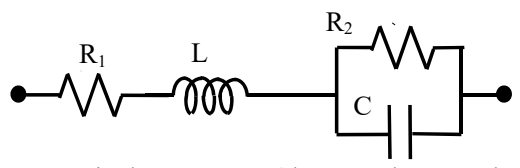

Figura 2. Circuito RL equivalente para resíduos e polos complexos conjugado.

Neste caso os elementos de circuito R, L e C podem ser calculados por (13)-(16).

$$
\begin{aligned}
& L=\frac{1}{\left(c_{1}+c_{2}\right)} \\
& C=\frac{\left(c_{1}+c_{2}\right)}{a_{1} a_{2}+k Q} \\
& R_{1}=\frac{K}{\left(c_{1}+c_{2}\right)}
\end{aligned}
$$




$$
R_{2}=-\frac{1}{C Q}
$$

Onde: $k=\left[-\left(a_{1}+a_{2}\right)+\left(\frac{c_{1} a_{2}+c_{2} a_{1}}{c_{1}+c_{2}}\right)\right]$ e $Q=\frac{c_{1} a_{2}+c_{2} a_{1}}{c_{1}+c_{2}}$

Fazendo em (8) os termos D e E iguais a zero, a curva da admitância será ajustada por frações parciais (último termo da expressão) que podem ser reais, complexas ou ambas e o circuito elétrico RLC equivalente resultante depende do tipo e quantidade dessas frações.

\section{RESULTADOS}

Embora para malha de aterramento pode se aplicar conceito similar para representá-la por circuitos elétricos, visto que a técnica proposta parte da impedância harmônica já determinada por um modelo prévio qualquer, neste trabalho foram apresentados resultados apenas para eletrodos horizontais e hastes verticais, conforme esquema mostrado na Fig. 3.

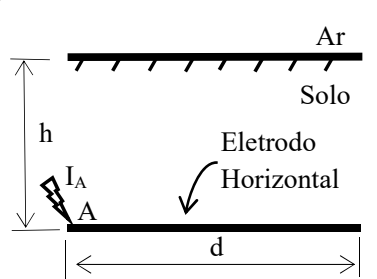

(a)

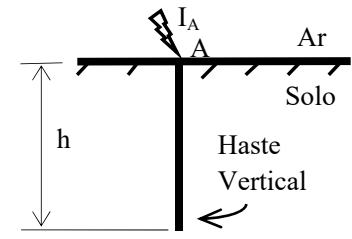

(b)
Figura 3. Esquema representativo do aterramento (a) para eletrodo horizontal e (b) para haste vertical.

O impulso atmosférico $\left(\mathrm{I}_{\mathrm{A}}\right)$ aplicado foi representado por uma onda de frente rápida, tipicamente $1,8 / 20 \mu \mathrm{s}$, com pico de $1.000 \mathrm{~A}$, matematicamente expressa por uma função dupla exponencial, injetada em uma das extremidades (ponto A) do eletrodo/haste, ver Fig. 3. Como a técnica proposta se aplica para representação da impedância do aterramento, ela não depende do tipo do impulso de corrente, podendo ser aplicada qualquer forma de onda de corrente para se obter a resposta transitória de tensão.

Os resultados foram obtidos percorrendo as seguintes etapas:

(i) Por meio do modelo baseado na teoria de linhas de transmissão, a partir de (7), se obteve a admitância harmônica equivalente ao aterramento mostrado na Fig. 3;

(ii) De posse da admitância aplicou-se o impulso de corrente $\left(\mathrm{I}_{\mathrm{A}}\right)$ para se obter, após conversão para o domínio do tempo, a forma de onda da elevação da tensão $\left(\mathrm{V}_{1}\right)$ no ponto de injeção da corrente. Ver Fig. 5 e Fig. 8. Essa forma de onda foi adotada como padrão para validação da técnica proposta;

(iii) Por meio de aproximação, usando-se o vector fitting, se determinou as funções racionais (8) que representam a admitância (módulo e fase) do aterramento de $10 \mathrm{~Hz}$ a $1 \mathrm{MHz}$;

(iv) A partir das funções racionais se obteve o circuito elétrico equivalente ao aterramento, ver Fig. 4 e Fig. 7, bem como os valores dos elementos discretos $\mathrm{R}, \mathrm{L}$ e $\mathrm{C}$ do circuito;

(v) De posse do circuito elétrico equivalente, aplicou-se um impulso de corrente $\mathrm{I}_{\mathrm{A}}$ (no domínio do tempo) e se obteve a forma de onda da elevação da tensão $\left(\mathrm{V}_{2}\right)$ no ponto $(\mathrm{A}) \mathrm{de}$ injeção da corrente. Ver Fig. 4 e Fig. 7; (vi) As formas de ondas das tensões $\mathrm{V}_{1}$ (considerada como padrão e obtida pelo modelo de LT) e $\mathrm{V}_{2}$ (obtida pelo circuito proposto que representa o aterramento) foram comparadas, ver Fig. 5 e Fig. 8, para validação da técnica proposta.

$\mathrm{O}$ circuito elétrico foi simulado no $\mathrm{MATLAB}^{\circledR}$, no domínio da frequência e a resposta foi convertida para o domínio do tempo e comparada com simulação no PSPICE ${ }^{\circledR}$ (ORCAD), diretamente no domínio do tempo. Os resultados, como esperado, foram praticamente os mesmos.

Para simulação de eletrodo horizontal foi considerado o comprimento de $10 \mathrm{~m}$, raio igual a $4 \mathrm{~mm}$, enterrado a uma profundidade de $0,7 \mathrm{~m}$ em solo com permissividade elétrica relativa igual a 15 , consideradas resistividades do solo de 100 , 500 e $1000 \Omega \mathrm{m}$ e frequências de $10 \mathrm{~Hz}$ a $1 \mathrm{MHz}$.

Seguindo as etapas (i) e (iii), as funções racionais (17), (18) e (19) foram obtidas para resistividades do solo de 100, $500 \mathrm{e}$ $1000 \Omega \mathrm{m}$, respectivamente.

$$
\begin{gathered}
Y(s)=\frac{4,5328 \cdot 10^{6}}{s-\left(-3,5394 \cdot 10^{8}\right)} \\
+\frac{0,2313 \cdot 10^{6}}{s-\left(-0,0416 \cdot 10^{8}\right)} \\
Y(s)=\frac{(2,3963-\mathrm{j} 0,1168) 10^{5}}{s-(-1,1996+\mathrm{j} 1,7486) 10^{7}} \\
\quad+\frac{(2,3963+\mathrm{j} 0,1168) 10^{5}}{s-(-1,1996-\mathrm{j} 1,7486) 10^{7}} \\
Y(s)=\frac{(1,7789-\mathrm{j} 0,2845) 10^{5}}{s-(-0,4372+\mathrm{j} 1,9164) 10^{7}} \\
+\frac{(1,7789+\mathrm{j} 0,2845) 10^{5}}{s-(-0,4372-\mathrm{j} 1,9164) 10^{7}}
\end{gathered}
$$

Os circuitos equivalentes a este sistema de aterramento (eletrodo horizontal) foram determinados a partir das funções racionais, conforme (iv) e, para resistividade do solo de $100 \Omega \mathrm{m}$, estão representados na Fig. 4a cujos elementos de circuitos são $\mathrm{L}_{1}=220,62 \eta \mathrm{H}, \quad \mathrm{R}_{1}=78,09 \Omega, \quad \mathrm{L}_{2}=4,32 \mu \mathrm{H}$, $\mathrm{R}_{2}=17.9648$. Para resistividades de $500 \Omega \mathrm{m}$ e $1000 \Omega \mathrm{m}$, ver Fig. 4b, cujos elementos são, respectivamente:

$\mathrm{L}_{1}=2,09 \mu \mathrm{H}, \mathrm{C}=1,56 \eta \mathrm{F}, \mathrm{R}_{1}=23,25 \Omega, \mathrm{R}_{2}=49,77 \Omega \mathrm{e}$

$\mathrm{L}_{1}=2,81 \mu \mathrm{H}, \mathrm{C}=944,54 \rho \mathrm{F}, \mathrm{R}_{1}=3,67 \Omega, \mathrm{R}_{2}=142,37 \Omega$.
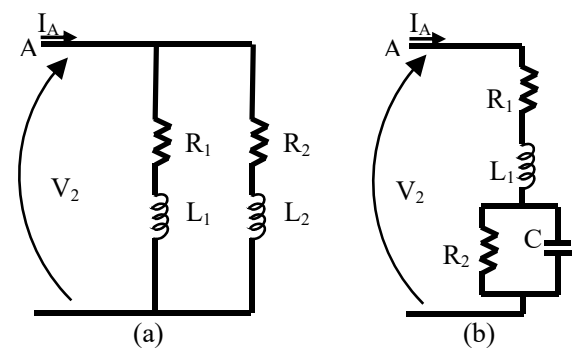

Figura 4. Circuitos equivalentes aos aterramentos, para solos de resistividade iguais a (a) $100 \Omega \mathrm{m}$ e (b) $500 \Omega \mathrm{m}$ e $1000 \Omega \mathrm{m}$.

Verifica-se que para representar o eletrodo de $10 \mathrm{~m}$ de comprimento em solo de baixa resistividade $(100 \Omega \mathrm{m})$ bastaram dois polos reais, mas em solos de media $(500 \Omega \mathrm{m})$ e alta $(1000 \Omega \mathrm{m})$ resistividade se fez necessário um par de polos complexos conjugados.

Com o propósito de mostrar que o circuito elétrico equivalente ao aterramento produz o mesmo resultado que sua 
admitância determinada pelo modelo de LT (7), foram apresentadas na Fig. 5 as formas de ondas das tensões transitórias $\mathrm{V}_{1}$ e $\mathrm{V}_{2}$, no ponto de injeção do impulso de corrente. As tensões identificadas por $\mathrm{V}_{1}$ foram obtidas via modelo de LT, conforme (ii) e as identificadas por $\mathrm{V}_{2}$ foram obtidas pelo circuito equivalente, conforme (v). Ambas para um eletrodo horizontal de $10 \mathrm{~m}$ de comprimento enterrado em

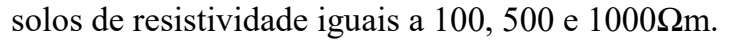

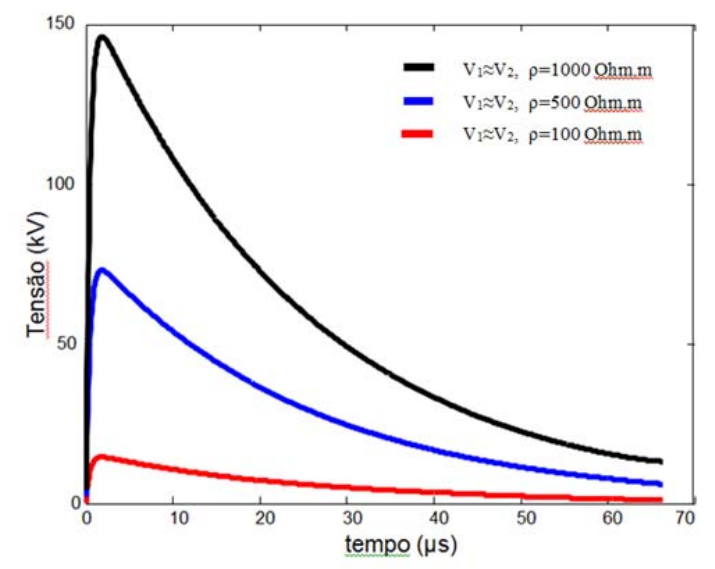

Figura 5. Elevação de tensão - eletrodo horizontal. Curvas $V_{1}$ - Padrão via modelo LT, Curvas $\mathrm{V}_{2}$ - via circuito RLC proposto.

Conforme (vi), comparando-se as tensões $\mathrm{V}_{1}$ e $\mathrm{V}_{2}$ verificase que elas são praticamente coincidentes provando que os circuitos elétricos, ver Fig. 4, determinados pela técnica proposta são capazes de representar com muita fidelidade os sistemas de aterramentos.

As diferenças entre os valores de pico ocorrem em função das diferentes resistividades adotadas para o solo. Quanto maior a resistividade do solo, maior a elevação de tensão, para um mesmo comprimento de eletrodo.

No caso de haste vertical o procedimento seguido é similar ao descrito nas etapas (i) a (vi). Como a técnica foi desenvolvida tendo como base o modelo de linhas de transmissão, a diferença no calculo da admitância de hastes verticais e de eletrodos horizontais está na forma de se calcular os parâmetros longitudinais e transversais da haste e do eletrodo ( $\mathrm{R}, \mathrm{L}, \mathrm{G}$ e $\mathrm{C})$, mas quanto à técnica desenvolvida para representá-los por meio de circuito discreto, nada se altera (ela parte da admitância já determinada).

Para simulação de hastes verticais foram consideradas hastes de 5, 10, 15 e $24 \mathrm{~m}$ de comprimento, com raio igual a $12,5 \mathrm{~mm}$, cravadas totalmente em solo de resistividade igual a

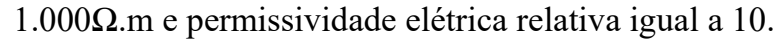

Para representá-las por circuitos elétricos, primeiramente foram determinadas as admitâncias para cada uma das hastes (5, 10, 15 e $24 \mathrm{~m}$ de comprimento), conforme etapa (i). As curvas das admitâncias estão apresentadas na Fig. 6, identificadas por Y. De posse das admitâncias, usando a técnica de aproximação, conforme etapa (iii) foram ajustadas as curvas de tal forma a coincidirem com as curvas da admitância. Estas curvas ajustadas pela função racional estão identificadas na Fig. 6 por FR. A garantia de resultados precisos é realizar o ajuste da quantidade de polos de tal forma que as curvas Y e FR se sobreponham.
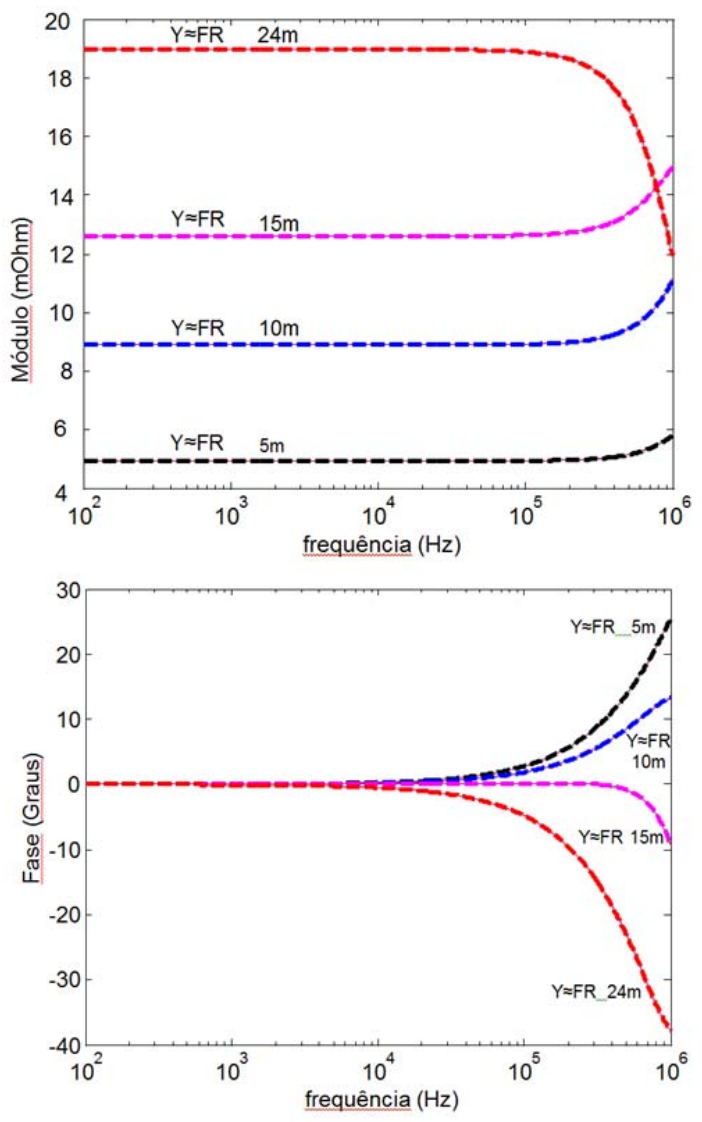

Figura 6. Ajuste das admitâncias (módulo e fase) por funções racionais.

Verifica-se o comportamento capacitivo (hastes de 5 e $10 \mathrm{~m}$ ) e o indutivo (hastes de 15 e $24 \mathrm{~m}$ ). Estas alterações de comportamento podem causar alterações nos circuitos representativos das hastes, ver Fig. 7.

$\mathrm{Na}$ sequência, a partir das funções racionais obtidas, foram encontrados os circuitos equivalentes a este sistema de aterramento (haste vertical), conforme (iv). Eles estão apresentados na Fig. 7 e os elementos RLC descritos a seguir: Haste de 5m: circuito equivalente da Fig. 7a, sendo $\mathrm{L}=1,78 \mu \mathrm{H}, \mathrm{C}=443,19 \mathrm{pF}, \mathrm{R}_{1}=2,25 \Omega, \mathrm{R}_{2}=200,76 \Omega$;

Haste de 10m: circuito equivalente da Fig. 7a, sendo $\mathrm{L}=3,29 \mu \mathrm{H}, \mathrm{C}=1,02 \mathrm{nF}, \mathrm{R}_{1}=18,57 \Omega, \mathrm{R}_{2}=93,97 \Omega$

Haste de 15m: circuito equivalente da Fig. 7a, sendo $\mathrm{L}_{1 \mathrm{a}}=4,06 \mu \mathrm{H}, \mathrm{R}_{1 \mathrm{a}}=47,43 \Omega, \mathrm{C}_{1 \mathrm{a}}=4,20 \mathrm{nF}, \mathrm{R}_{2 \mathrm{a}}=31,93 \Omega$

Haste de 24m: circuito equivalente da Fig. 7b, sendo $\mathrm{L}_{1 \mathrm{a}}=15,96 \mu \mathrm{H}, \quad \mathrm{R}_{1 \mathrm{a}}=6,39 \Omega, \quad \mathrm{C}_{1 \mathrm{a}}=1,64 \mathrm{nF}, \quad \mathrm{R}_{2 \mathrm{a}}=55,45 \Omega \quad$ e $\mathrm{L}_{1 \mathrm{~b}}=1,40 \mu \mathrm{H}, \mathrm{R}_{1 \mathrm{~b}}=88,83 \Omega, \mathrm{C}_{1 \mathrm{~b}}=647,33 \mathrm{pF}, \mathrm{R}_{2 \mathrm{~b}}=267,66 \Omega$.

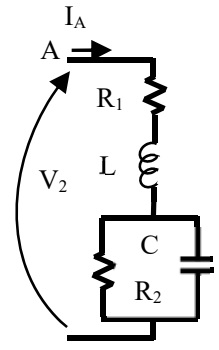

(a)

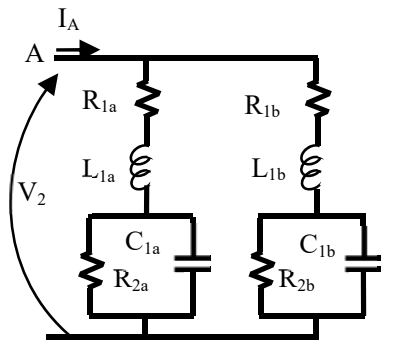

(b)
Figura 7. Circuitos equivalentes aos aterramentos (a) haste de 5,10 e $15 \mathrm{~m}$ de comprimento e (b) haste de $24 \mathrm{~m}$ de comprimento. 
Verifica-se que para os comprimentos de hastes de 5, 10 e $15 \mathrm{~m}$ foi possível representá-las por uma função com um par de polos complexos conjugados, ver Fig. 7a, e que para comprimento de $24 \mathrm{~m}$ se fez necessário dois pares, ver Fig. $7 \mathrm{~b}$.

Com o propósito de mostrar que o circuito elétrico equivalente ao aterramento produz o mesmo resultado que sua admitância determinada pelo modelo de LT (7), foram apresentadas na Fig. 8, as formas de ondas das tensões transitórias $\mathrm{V}_{1}$ e $\mathrm{V}_{2}$, no ponto de injeção do impulso de corrente. As tensões identificadas por $\mathrm{V}_{1}$ foram obtidas via modelo de LT, conforme (ii) e as identificadas por $\mathrm{V}_{2}$ foram obtidas pelo circuito equivalente, conforme (v).

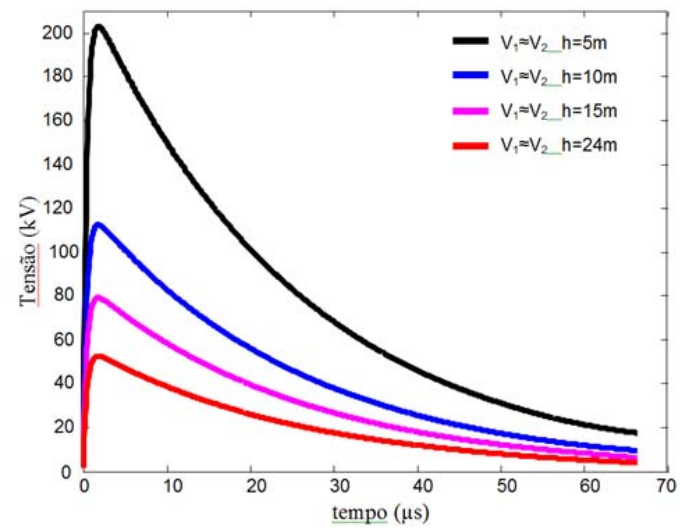

Figura 8. Elevação de tensão - haste vertical. Curvas $\mathrm{V}_{1}$ - Padrão (modelo LT), Curvas $\mathrm{V}_{2}$ - modelo proposto RLC.

Conforme (vi), comparando-se as tensões $\mathrm{V}_{1}$ e $\mathrm{V}_{2}$ verificase que elas são praticamente coincidentes, provando que os circuitos propostos na Fig. 7 são capazes de representar com fidelidade o sistema de aterramento composto por uma haste vertical.

Para validação dos resultados, além da comparação entre as tensões $V_{1}$ e $V_{2}$, também foram comparados os resultados obtidos para haste de $24 \mathrm{~m}$ com os resultados obtidos por Grcev [15], que usou outra técnica de aproximação aplicada à curva da impedância harmônica obtida por ele, via modelo eletromagnético (EM). Na Fig. 9 é apresentado o circuito elétrico equivalente à haste vertical de $24 \mathrm{~m}$ citada, conforme modelo de Grcev.

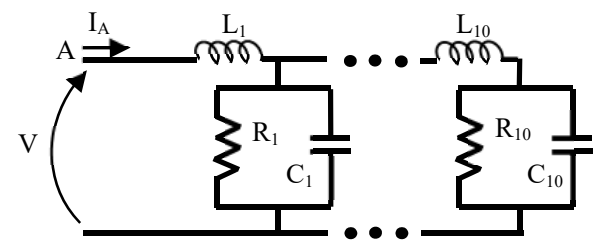

Figura 9. Circuito equivalente à haste de $24 \mathrm{~m}$ via modelo Grcev.

Naquele modelo, uma das propostas do autor era melhorar o ajuste da curva da impedância harmônica obtida pelo modelo de linhas de transmissão com circuitos $(\pi)$ uniformemente distribuídos, comparada com a referência obtida pelo modelo eletromagnético. Ele realizou este ajuste transformando os circuitos uniformemente distribuídos em circuitos não uniformemente distribuídos, fixando os valores das resistências e capacitâncias de todas as seções concentradas do circuito e calculando os valores das indutâncias, via rotina específica no MATLAB ${ }^{\circledR}$ para obter um melhor ajuste entre as curvas. Os elementos calculados foram: $\mathrm{L}_{1}$ a $\mathrm{L}_{10}(\mathrm{em} \mu \mathrm{H})$ igual a 1,92; 6,$73 ; 4,36 ; 4,41 ; 3,95 ; 3,47 ; 3,15 ; 2,59 ; 2 ; 0,36 ; \mathrm{R}_{1}$ a $\mathrm{R}_{10}=527 \Omega$ e $_{1}$ a $\mathrm{C}_{10}=168 \mathrm{pF}$.

Na Fig. 10 é mostrado o resultado da simulação no domínio do tempo (PSPICE) para o circuito da Fig. 7b e o da Fig. 9, onde é possível observar boa concordância entre as curvas, permitindo concluir que nas condições simulada o circuito sugerido na Fig. $7 \mathrm{~b}$ pode ser substituído pelo sugerido na Fig. 9 e vice versa.

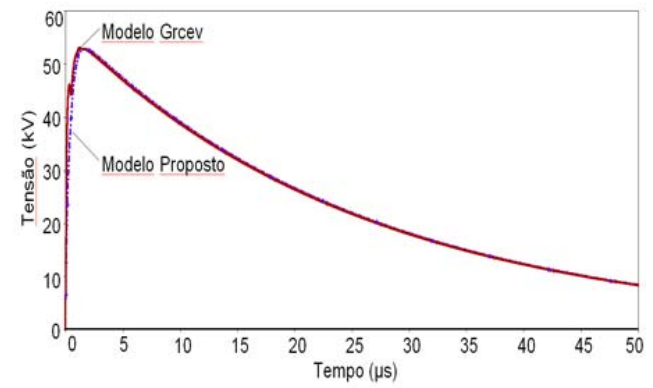

Figura 10. Comparação dos resultados do circuito da Fig. 7b e da Fig. 9.

No modelo proposto, a topologia do circuito representativo da haste depende da quantidade de frações parciais geradas na aproximação e dos seus tipos (reais ou complexas). Ela não é previamente definida como no modelo apresentado por Grcev, pois para cada resistividade/permissividade de solo ou comprimento de haste poderá resultar em circuitos elétricos equivalentes diferentes. A ligeira diferença entre as curvas de tensão pode ser devido às curvas da admitância não serem exatamente iguais (determinadas via modelos de LT e EM).

Muitos dos eletrodos horizontais ou hastes verticais usados em aterramento podem ser representados por circuitos equivalentes similares aos mostrados nas Fig. 11, isto é, por um até seis polos reais (Fig. 11a), por um polo real e dois polos complexos (Fig. 11b), por quatro polos complexos (Fig. 11c) e por um polo real e quatro polos complexos (Fig. 11d). A topologia de circuito vai depender basicamente da resistividade e permissividade do solo e do comprimento e raio do eletrodo/haste.

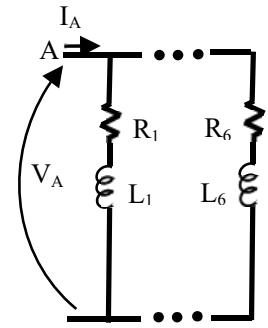

(a)

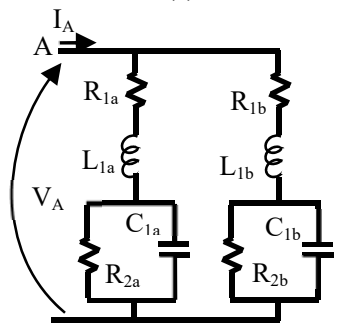

(c)

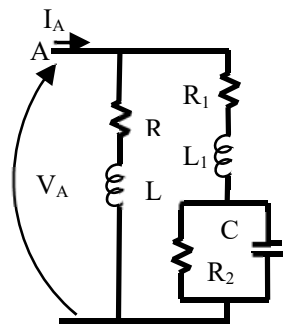

(b)

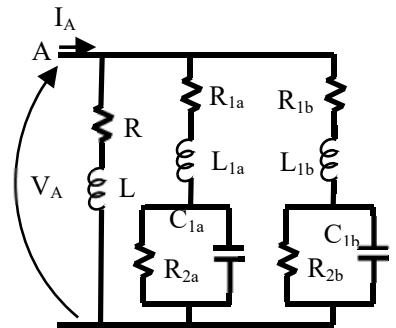

(d)
Figura 11. Circuitos representativos de aterramentos. 
Sabe-se que em altas frequências, entre $1 \mathrm{M}$ e $10 \mathrm{Mhz}$, o comportamento do eletrodo ou haste de aterramento pode alternar entre capacitivo e indutivo, ocasionando oscilações na curva da impedância. Para representação do aterramento nessas frequências, circuitos com maior quantidade de resíduos/polos poderão ser exigidos. Porém, é usual no estudo de aterramentos, devido as frequências típicas das descargas atmosféricas de retorno (primeira descarga), representações até 1 ou $2 \mathrm{MHz}$. Geralmente o tipo de aplicação define a faixa de frequência adequada para estudo.

\section{CONCLUSÃO}

Esse trabalho apresentou uma técnica capaz de representar um sistema de aterramento, diretamente no DT, por elementos discretos de circuitos elétricos. Consiste em uma nova ferramenta para análise de transitórios eletromagnéticos em sistemas de aterramento elétrico.

A técnica se mostrou bastante precisa e não requer grandes recursos computacionais. Apresenta como grande vantagem permitir que simulações sejam realizadas diretamente no DT pela maioria dos softwares de simulação disponíveis no mercado (exemplos: ATP, EMTP e PSPICE). Permite também a associação com outros elementos de circuito, como por exemplo, o modelo de uma torre de transmissão, para se fazer a análise transitória do conjunto.

Os modelos apresentados na Fig. 11 atendem diversos comprimentos de eletrodo e diferentes resistividades de solo, porém podem existir situações particulares que necessitem de algum ajuste. No entanto, um mesmo aterramento pode ser representado por infinitos circuitos diferentes.

Para análise transitória de um sistema real de aterramento, aplicando-se a técnica proposta neste trabalho, é necessário conhecer a sua impedância harmônica e disponibilizar de softwares de simulação (ex.: MATLAB®).

Este trabalho abordou somente eletrodo horizontal e haste vertical de aterramento. No entanto, conceito similar pode ser empregado para outras geometrias de aterramento como, por exemplo, malhas e anéis, requerendo apenas que seja fornecida a impedância harmônica de tal geometria.

A técnica proposta não depende do modelo utilizado para determinar a impedância harmônica nem do tipo de impulso de corrente injetado no aterramento. Ela representa o aterramento por meio de circuitos elétricos, possibilitando análises diretamente no domínio do tempo, dispensando o uso de transformada inversa de Laplace ou Fourier. Porém a precisão da análise dependerá do modelo e suas aproximações usadas para determinação da impedância harmônica.

\section{AGRADECIMENTOS}

Ao Instituto Federal de São Paulo (IFSP) e à Universidade Estadual Paulista (UNESP), Campus de Ilha Solteira.

\section{REFERÊNCIAS}

[1] Y. Liu, M. Zitnik and R. Thottappillil, "An improved transmission-line model of grounding system," IEEE Trans. Electromagnetic Compatibility, vol. 43, no. 3, pp. 348-355, Aug. 2001.

[2] L. Grcev, "Computation of transient voltages near complex grounding systems caused by lightning currents", IEEE 1992. International Symposium on Electromagnetic Compatibility, Anaheim, CA, pp. 393-400, 1992.
[3] R. Verma and D. Mukhedkar, "Impulse impedance of buried ground wire,” IEEE Trans. Power App. Syst., vol. 99, pp. 2003-2007, Sep./Oct. 1980. [4] J. Cidras, A. F. Otero and C. Garrido, "Nodal frequency analysis of grounding systems considering the soil ionization effect," IEEE Trans. Power Del., vol. 15, no. 1, pp. 103-107, Jan. 2000.

[5] R. Velazquez and D. Mukhedkar, "Analytical modeling of grounding electrodes," IEEE Trans. Power App. Syst., vol. PAS-103, no. 6, pp. 1314 1322, Jun. 1984.

[6] Y. Liu, N. Theethayi and R. Thottappillil, "An engineering model for transient analysis of grounding system under lightning strikes: Nonuniform transmission-line approach," IEEE Trans. Power Del., vol. 20, no.2, pp. 722 730, Apr. 2005

[7] L. Grcev, "Computer analysis of transient voltages in large grounding systems," IEEE Trans. Power Del., vol. 11, no. 2, pp. 815-823, Apr. 1996.

[8] S. Visacro. "A comprehensive approach to the grounding response to lightning currents". IEEE Transactions on Power Delivery, vol. 22, n. 1, pp. 381-386, Jan. 2007.

[9] L. V. Bewley. "Theory and tests of the counterpoise", AIEE Transactions, vol. 53, pp. 1163-1172, Aug 1934.

[10] R. Rudenburg, "Grounding principles and practice I - Fundamental considerations on ground circuits", Electrical Engineering, vol. 64, no. 1, pp. 1-13, Jan 1945.

[11] E. D. Sunde, "Earth conduction effects in transmission systems", 2nd ed. New York: Dover, 1968.

[12] B. R. Gupta and B. Thapar, "Impulse impedance of grounding grids", IEEE Transactions on Power Apparatus and Systems, vol.PAS-99, no.6, pp. 2357-2362, Nov./Dec. 1980.

[13] R. Olsen and M. C. Willis, "A comparison of exact and quasi-static methods for evaluating grounding systems at high frequencies," IEEE Trans. Power Del., vol. 11, no. 3, pp. 1071-1081, Jul. 1996.

[14] L. Grcev and S. Grceva, "On HF circuit models of horizontal grounding electrodes", IEEE Trans. Electromagn. Compat., vol. 51, no. 3, pp. 873-875, Aug. 2009.

[15] L. Grcev and M. Popov, "On high-frequency circuit equivalents of a vertical ground rod," IEEE Trans. Power Del., vol. 20, no. 2, pp. 1598-1603, Apr. 2005.

[16] A. Budner. "Introduction of Frequency-Dependent Line Parameters into an Electromagnetic Transients Program". IEEE Trans. on Power Apparatus and Systems, vol. 89, no 1, pp 88-97, Jan. 1970

[17] B. Gustavsen and A. Semlyen, "Rational approximation of frequency domain responses by Vector Fitting", IEEE Trans. Power Delivery, vol. 14, no. 3, pp. 1052-1061, Jul. 1999.

[18] G. Antonini, "SPICE equivalent circuit of frequency-domain Responses", IEEE transactions on electromagnetic compatibility, vol. 45, no. 3, Aug. 2003.

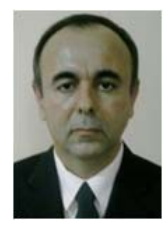

Claudiner Mendes de Seixas, graduado em engenharia elétrica (1991), obteve o título de Mestre em Engenharia Elétrica (1993) pela Universidade Federal de Santa Catarina (UFSC). A partir de 1993 trabalhou 16 anos na área de infraestrutura para Telecomunicações. Recebeu o título de Especialista em Gestão Empresarial (2000) pela Universidade Estadual de Maringá (UEM). É professor do Instituto Federal de São Paulo (IFSP) desde 2011 e aluno do curso de pós graduação (doutorado) da Universidade Estadual Paulista (UNESP), Campus Ilha Solteira. Suas áreas de interesse incluem sistemas de aterramento elétrico, transitórios eletromagnéticos, qualidade de energia, aplicações utilizando energia limpa.

http://lattes.cnpq.br/2837838465138774

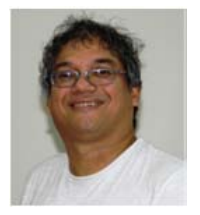

Sérgio Kurokawa, graduado em Engenharia Elétrica (1990). Desde 1994 atua como Professor na Faculdade de Engenharia de Ilha Solteira da Universidade Estadual Paulista (UNESP). Obteve o título de Doutor em Engenharia Elétrica (2003) na Faculdade de Engenharia Elétrica e da Computação da Universidade Estadual de Campinas (UNICAMP). Suas principais áreas de interesse são transitórios eletromagnéticos em sistemas elétricos de potência e modelos de linhas de transmissão para simulações de transitórios eletromagnéticos em sistemas de potência.

http://lattes.cnpq.br/4830845230549223 